Check for updates

Cite this: Analyst, 2018, 143, 5487

\title{
Qualitative analysis of antibody-drug conjugates (ADCs): an experimental comparison of analytical techniques of cysteine-linked ADCs $\uparrow$
}

\author{
Malin Källsten, ${ }^{* a, c}$ Rafael Hartmann, (D) ${ }^{b}$ Konstantin Artemenko, ${ }^{a}$ \\ Sara Bergström Lind, (D) ${ }^{a}$ Fredrik Lehmann ${ }^{c}$ and Jonas Bergquist (DD *a
}

\begin{abstract}
Antibody-drug conjugates (ADCs) are an emerging type of biotherapeutics that utilize multiple tissuespecific antibodies combined with a range of linker designs to enable the transportation and selective release of cytotoxic drugs in close proximity to tumours. Consisting of antibodies conjugated to small drug molecules through a variety of linkers, ADCs are chemically complex analytes. Here we present a unique experimental comparison of four techniques for ADC analysis: hydrophobic interaction chromatography (HIC-UV/Vis), reversed phase liquid chromatography mass spectrometry (RPLC-MS), using either a QToF or an Orbitrap analyser, and matrix-assisted laser desorption ionization time-of-flight mass spectrometry (MALDI-TOF-MS). Four different ADCs consisting of Trastuzumab, monomethyl auristatin E (MMAE) and a peptidic linker moiety differing in their respective stoichiometric ratios in regard to drugto-antibody ratio (DAR) were used for the comparison. We found that the determined DAR from all techniques was comparable, while the accuracy of the molecular weights for the conjugated light and heavy chain differed more extensively. This indicates that the choice of a mass analyser is more crucial for determining the accurate weights of the light and heavy chains than to evaluate the DAR of a given batch. However, ambiguous DAR assignment in HIC-UV/Vis or bias for either the light or heavy chain fragments in the mass spectrometry-based techniques can influence the obtained average DAR value and the use of complementary techniques is advisable. Out of the four techniques evaluated, HIC-UV/Vis and MALDI required less time to obtain an average DAR value and would therefore be good for initial screenings in the early stages of the discovery phase of new ADCs.
\end{abstract}

Received 27th June 2018, Accepted 6th September 2018

DOI: 10.1039/c8an01178h

rsc.li/analyst in breast cancer) or overexpressed at a certain stage of disease, the systemic toxicity of the administered drug is reduced. The drug release outside the intended target can thus be limited and the efficacy is further increased by the selection of a chemical linker (cleavable or non-cleavable) with ideal pharmacokinetic properties. ${ }^{5}$ Additional complexity originates from the lack of regioselectivity associated with the conjugation to native amino acid sites, such as the cysteines or the lysines in the primary protein structure. Apart from deliberate modifications of the antibody structure during ADC production, all naturally produced antibodies also have several inherited post-translational modifications (PTMs). The most common PTMs include glycosylation, deamidation and phosphorylation. PTMs commonly alter between production batches and changes may profoundly impact protein aggregation, lead to varying degrees of immunogenicity ${ }^{6}$ upon administration or impact the efficacy. ${ }^{7-9}$

As a means of risk-mitigation, the FDA has placed a high demand on the analytical characterization of all monoclonal antibody-based biotherapeutics ${ }^{10,11}$ including ADCs. Hence, there is a dire need for reliable analytical techniques to meet 
those requirements. Whilst much work has been done to control the regioselectivity of the covalent attachment of drug molecules onto the antibodies and new modes of drugrelease, ${ }^{12-15}$ little attention has been paid to ADC-analyses until recently. Recent publications described both novel techniques for the assessment of the drug-to-antibody ratio (DAR), ${ }^{16-21}$ ADC stability, ${ }^{2-25}$ aggregation degree assessment, ${ }^{26-28}$ bioanalysis, ${ }^{29}$ the quantification of unconjugated drug molecules ${ }^{22}$ and PTMs. 8 ,30-32

There exist numerous reviews discussing ADC analysis techniques by comparing data from different studies; ${ }^{27,33-35}$ however, to our knowledge comprehensive studies that have experimentally compared the performance of multiple techniques for ADC DAR analysis based on a single sample batch are scarce. ${ }^{16,36,37}$ This study aims to experimentally compare commonly applied analytical techniques for DAR analysis, a key characteristic of individual ADC-batches. Herein we have analysed four ADCs with distinctively different average DARs based on Trastuzumab and cysteine-linked MMAE with a peptidase cleavable linker using HIC-UV/Vis, RPLC-MS (QToF and Orbitrap) and MALDI-TOF-MS. This is a unique comparison of an identical set of samples analysed by four different techniques providing information on their respective performance with focus on DAR determination. The selection was largely based on the most prevalent techniques in the literature for DAR determination of cysteine-linked ADCs. MALDI-TOF-MS has not been utilized for DAR determination before but was included due to its prospect to be used in fast screening since neither deconvolution nor HPLC-separation is needed as opposed to RPLC-MS. It also enables screening for artefacts in the ESI-MS deconvoluted spectra and unaccounted losses of certain DAR species on-column during the RPLC separation.

\section{Experimental}

\section{Chemicals}

All chemicals were purchased from Sigma-Aldrich with the following exceptions: acetonitrile (ACN) and formic acid (FA) for the Orbitrap analyses (Thermo Scientific), Trastuzumab (Carbosynth Ltd), dithiothreitol (DTT) used for reduction prior to analysis (Panreac Applichem) and VcMMAE (abcr GmbH).

\section{Samples}

Trastuzumab-VcMMAE-conjugates with different degrees of cysteine conjugation (low, medium, high and maximum) were prepared in accordance with an established procedure, ${ }^{38}$ with some modifications. Briefly, "low"- and "mid"-DAR species were generated by partial reduction of interchain disulphides, followed by an addition of an excess of linker-drug reagent, while "high"- and "max"-DAR species were generated from fully reduced antibodies.

A solution of antibody in borate buffer $(50 \mathrm{mM}$ borate, $60 \mathrm{mM}$ chloride, $\mathrm{pH}$ 8.0) was prepared and treated with DTT (2.25 eq. for "low"-DAR and 3.25 eq. for "mid"-DAR, $10 \mathrm{mM}$ final concentration for complete disulphide reduction) to a concentration of $1 \mathrm{mg} \mathrm{mL} \mathrm{m}^{-1}$ of protein. After 30 minutes of incubation at $37{ }^{\circ} \mathrm{C}$, the reduced antibodies were desalted (Amicon Ultra-4, $30 \mathrm{kDa}$ MWCO), taken up in PBS containing pentetic acid (DTPA) (10 mM phosphate, $140 \mathrm{mM}$ chloride, $1 \mathrm{mM}$ DTPA) and diluted with a solution of VcMMAE in ACN to a protein concentration of $2 \mathrm{mg} \mathrm{mL}^{-1}$ and a total of $20 \%$ organic solvent. 13 molar equivalents of VcMMAE were added to the partially reduced antibodies, while 5 and 11 molar equivalents were added to generate "high"- and "max"-DAR species, respectively. Upon incubation at $4{ }^{\circ} \mathrm{C}$ for 2 hours, the reaction mixtures were desalted once again, diluted to a concentration of $1 \mathrm{mg} \mathrm{mL}^{-1}$ using PBS (10 mM phosphate, $140 \mathrm{mM}$ chloride) and aliquoted into $200 \mu \mathrm{g}$ portions (1 mg $\mathrm{mL}^{-1}$ in $10 \mathrm{mM}$ PBS for all except the "low" DAR which had only $0.67 \mathrm{mg} \mathrm{mL}^{-1}$ ) before any further sample preparation.

$42 \mu \mathrm{g}$ of each batch was deglycosylated by adding $2 \mu \mathrm{L}$ of PNGaseF (500 units per $\mathrm{mL}$ ) at $37{ }^{\circ} \mathrm{C}$ and incubated for 3 hours. The interchain disulphide bonds were then reduced by addition of $4 \mu \mathrm{L}$ of $1 \mathrm{M}$ DTT, followed by incubation at room temperature for $30 \mathrm{~min}$. The samples were diluted to a final concentration of $0.5 \mathrm{mg} \mathrm{mL} \mathrm{m}^{-1}$ in $15 \% \mathrm{ACN}$ and $0.1 \% \mathrm{FA}$ and stored at $-20{ }^{\circ} \mathrm{C}$ until analysis.

\section{HIC-UV}

$80 \mu \mathrm{g}$ of protein was diluted to a final concentration of $0.8 \mathrm{mg} \mathrm{mL} \mathrm{m}^{-1}$ in $1 \mathrm{M}$ ammonium sulphate before analysis. HIC-UV/Vis was performed using Waters Protein Pak Hi Res HIC $2.5 \mu \mathrm{m} 4.6 \times 100 \mathrm{~mm}$ on an Agilent 1100 HPLC system with a diode-array detector monitoring the signal at $220 \mathrm{~nm}$ and $280 \mathrm{~nm}$. Gradient elution was performed with $0-100 \%$ mobile phase B (62.5 mM sodium phosphate buffer ( $\mathrm{pH}$ 6.9) with 5\% isopropanol), while mobile phase A consisted of $62.5 \mathrm{mM}$ sodium phosphate buffer ( $\mathrm{pH}$ 6.9), 5\% isopropanol and $1.25 \mathrm{M}\left(\mathrm{NH}_{4}\right)_{2} \mathrm{SO}_{4} .16 \mu \mathrm{g}$ of sample was injected in triplicate with three blank runs between each sample set. The gradient started at $0 \% \mathrm{~B}$ and linearly increased to $100 \% \mathrm{~B}$ over $10 \mathrm{~min}$ before reverting to $0 \% \mathrm{~B}$ (over $0.1 \mathrm{~min}$ ) and finally reconditioning the column for $20 \mathrm{~min}$. The flow rate was $0.7 \mathrm{~mL} \mathrm{~min}^{-1}$.

\section{RPLC-MS}

RPLC-MS was performed using two different MS: Waters's Q-ToF Premier and Thermo's Orbitrap Q-Exactive Plus. The Q-ToF was coupled to a Waters Acquity UPLC system with an Agilents AdvanceBio RP-mAb C4 $2.1 \times 100 \mathrm{~mm}$ column (3.5 $\mu \mathrm{m}$ semi-porous particles with $450 \AA$ pores). Gradient elution was performed at $65{ }^{\circ} \mathrm{C}$ with $15-95 \%$ mobile phase $\mathrm{B}$ ( $0.1 \%$ FA in ACN), while mobile phase A constituted of $0.1 \%$ FA in water. $5 \mu \mathrm{g}$ of each sample was injected in triplicate with three blank runs between each sample set (injecting $0.1 \%$ FA). The gradient started at 15\% B for $1 \mathrm{~min}$ and was then linearly increased to $95 \%$ over $11 \mathrm{~min}$. It was kept at $95 \%$ for $1 \mathrm{~min}$ before going back to $15 \%$ (over $0.2 \mathrm{~min}$ ) and reconditioned for $2 \mathrm{~min}$. The flow rate was $0.4 \mathrm{~mL} \mathrm{~min}^{-1}$ and a solvent delay of 2 min was applied at the start of the MS spectra acquisition. The mass spectra were obtained in positive mode using single 
reflector mode in the $500-4500 \mathrm{~m} / \mathrm{z}$ range and a scan time of $1 \mathrm{~s}$ with an interscan delay of $0.1 \mathrm{~s} .800 \mathrm{~L} \mathrm{~h}^{-1}$ desolvation gas,

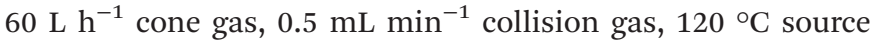
temperature and $300{ }^{\circ} \mathrm{C}$ desolvation temperature were used to help with the desolvation with a capillary voltage of $4.5 \mathrm{kV}$ and a cone voltage of $60 \mathrm{~V}$.

The Orbitrap was coupled to an Agilent 1260 Infinity Quaternary LC with a Thermo's Acclaim PepMap 300 C4 (5 $\mu \mathrm{m}$ semi-porous particles with $300 \AA$ pores) $1 \times 150 \mathrm{~mm}$ microcolumn. Gradient elution was performed at $60{ }^{\circ} \mathrm{C}$ (the highest temperature permitted for the column) with $20-95 \%$ mobile phase B (0.1\% FA in ACN), while mobile phase A consisted of $0.1 \%$ FA in water. $0.25 \mu \mathrm{g}$ of each sample was injected in triplicate with three blank runs between each sample set (injecting $0.1 \% \mathrm{FA})$. The gradient started at $20 \% \mathrm{~B}$ for $2 \mathrm{~min}$ and was then linearly increased to $95 \%$ over $17 \mathrm{~min}$. It was kept at $95 \%$ for $3 \mathrm{~min}$ before going back to $20 \%$ (over $2 \mathrm{~min}$ ) and reconditioned for $2 \mathrm{~min}$. The flow rate was $6 \mu \mathrm{L} \mathrm{min}^{-1}$. The mass spectra were obtained in full scan mode. The scan time was 10 microscans with the AGC target set to 1E6, in-source CID 70 $\mathrm{eV}$ and the maximum injection time of $200 \mathrm{~ms}$ for $\mathrm{m} / \mathrm{z}$ range $800-4000$ and a resolution of 35000 at $\mathrm{m} / \mathrm{z} 200$. The capillary temperature was $320^{\circ} \mathrm{C}$, the spray voltage was $4 \mathrm{kV}$ and the sheath gas flow was 8 units.

\section{MALDI-TOF-MS}

$0.5 \mu \mathrm{g}$ protein was deposited in triplicate on an Anchorchip target and covered with $1 \mu \mathrm{L}$ of $10 \mu \mathrm{g} \mu \mathrm{L}^{-1}$ Super-DHB (SDHB). Bruker's Autoflex II MALDI-TOF-MS calibrated externally using Bruker Protein Standard II ( $m / z 22300$ to $66500 \mathrm{Da})$ with a detection window of (20000-140000 Da) and $8.8 \times$ enhanced detector gain $(100 \mathrm{mV})$ with $0.5 \mathrm{GS}$ per s sample rate was used. Spectra are based on averaging 1400 shots on different positions with a frequency of $50 \mathrm{~Hz}$ on the same sample spot.

\section{Data evaluation}

Deconvolution was performed with Waters's Masslynx 4.1 with MaxEnt1 for the QToF data averaging the mass spectra from all peaks in the TIC (rt 3-6 min). Thermo's BioPharma Finder 2.0 software with the sliding window function was used for the Orbitrap data for the last peak observed in the TICs (rt 14-20 min). All DAR values were calculated using the peak area for the HIC and the peak intensity for the MS-techniques.

\section{Results and discussion}

The DAR of an ADC is defined as the number of drug molecules conjugated to a specific antibody. Generally, an average value is reported which directly determines the drug load delivered to the tumour cells per administered $\mu \mathrm{g}$ of ADC. In HIC-UV/Vis ADC-species are separated based on their $\mathrm{DAR}^{33,39,40}$ by the use of a decreasing salt gradient since increasing DAR generally corresponds to an increased hydrophobicity. HIC is generally only applicable for cysteine-linked ADCs since lysine linked ADCs commonly contain too many
DAR variants in one batch to be fully resolved chromatographically. RPLC-MS has the potential to spectrally resolve all DAR species of an $\mathrm{ADC}^{34,41}$ and MS-analysers eliminate the demand for complete chromatographic separation of different DAR species, simplifying the optimization process. RPLC-MS analysis of the DAR has traditionally been performed using QToF systems; however, recent years have seen more publications using Orbitrap mass analyzers. ${ }^{30,42}$ When performing animal studies or analysing the very first batches of different synthesis protocols, especially in the discovery phase, only limited amounts of samples are available. Hence MS-based techniques are favoured due to their lower detection limit. A convenient alternative to RPLC-MS is MALDI-TOF-MS ${ }^{43}$ since the singly charged ions produced make deconvolution obsolete. In later stages of drug development, time constraints are of bigger concern and it is valuable to have a quick analysis. HIC-UV/Vis or potentially MALDI would then be the optimal technique. New matrices such as Super DHB have improved the detection of larger proteins. There are a few publications utilizing MALDI for ADC analysis; however, it has yet to be evaluated as an option for DAR determination. ${ }^{44-47}$ Herein we have evaluated its suitability for DAR determination of reduced ADCs.

This experimental comparison of techniques was primarily concerned with the DAR determination. A single sample set of four cysteine-linked ADC batches with a distinct degree of conjugation was investigated with all four different techniques. The average DAR values reported in Table 1 corresponded considerably well between the techniques for the higher DAR batches. Furthermore, all techniques showed the smallest relative standard deviation for the DAR for the sample batch with the highest DAR (max. DAR). Thus, we observed that the choice of technique for DAR-determination is not as crucial for samples containing only a small number of DAR species. Below follows a more detailed evaluation of the investigated techniques.

\section{HIC-UV/Vis}

In the HIC comparison of the different ADC batches, the higher the conjugation degree, the harder it was to assign a specific DAR to a singular peak (see Fig. 1). Apart from the presence of odd numbered DAR species, ${ }^{25}$ extra peaks may also originate from the differences in PTMs, ${ }^{39,48}$ degradation products or positional isomers ${ }^{36}$ complicating the DAR evaluation. Early data in this study presented chromatograms with a large number of DAR variants for two of the ADC batches (Fig. 2, left panel). This prompted an alternation of the synthesis protocol. Switching from the use of an excess of

Table 1 Average DAR calculated from each technique in triplicate with RSD values in parenthesis

\begin{tabular}{lllll}
\hline & LOw DAR & Mid DAR & High DAR & Max. DAR \\
\hline HIC-UV/Vis & $1.0(1.9 \%)$ & $2.0(5.7 \%)$ & $6.1(1.2 \%)$ & $7.8(1.1 \%)$ \\
MALDI-TOF-MS & $1.1(13 \%)$ & $1.5(4.2 \%)$ & $6.0(1.7 \%)$ & $7.4(1.1 \%)$ \\
RPLC-MS (QToF) & $1.2(2.1 \%)$ & $1.6(5.1 \%)$ & $5.9(0.5 \%)$ & $7.0(0.8 \%)$ \\
RPLC-MS (Orbitrap) & $1.6(9.4 \%)$ & $2.2(18 \%)$ & $5.9(0.2 \%)$ & $7.9(0.5 \%)$
\end{tabular}




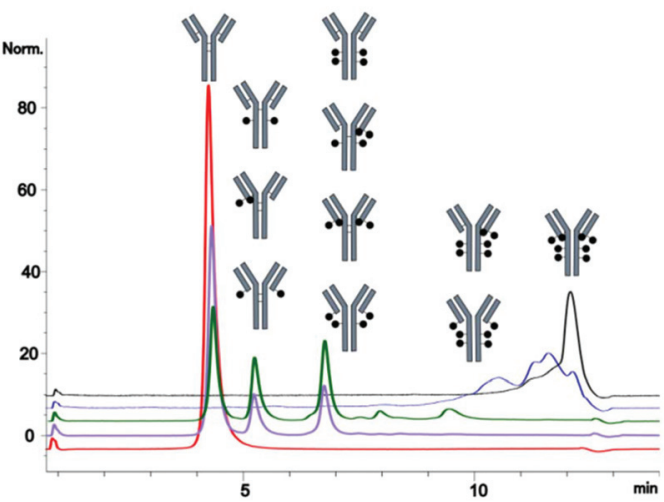

Fig. 1 HIC-UV/Vis chromatograms of DAR species for all five batches of samples: red (mAb), purple (low), green (Mid), blue (High) and black (Max), all corrected to the same scale.

reduction agent to an excess of the drug provided a much clearer chromatogram, as has also been discussed by Michael M. C. Sun et al. $^{49}$ This addition of complexity was however not detectable in any of the MS-based approaches under denaturing conditions. HIC was therefore concluded to be particularly suitable for initial screening in order to determine the success of a conjugation protocol, e.g. to ensure a limited range of subspecies before further verifications of the DAR species in each batch. HIC-UV/Vis did not however provide confirmation of the molecular weights and conjugation degree of the different chains of the antibodies, which we consider a clear disadvantage compared to the other analytical techniques.

HIC-UV/Vis required very little sample preparation and data evaluation time; however, due to the large drop in the baseline observed over the salt gradient, at least 10 minutes of re-equilibration was needed in between runs to ensure chromatographic reproducibility. The time addition from re-equilibration was partly compensated by the low number of blank runs needed between sample sets from the minimal carry over observed (ESI, Fig. S1-S15†). Despite the almost non-existent background interference, the drop in the baseline slightly increased the limit of detection and over $15 \mu \mathrm{g}$ of protein was needed in each injection to allow peak integration. The large heterogeneity inherited in ADCs requires at least partial optim- ization of each technique before a reliable DAR value can be determined for a completely new construct, thus setting up HIC-UV/Vis for each new ADC can consume a large portion of the sample available in the initial screening.

\section{RPLC-MS}

Orbitrap MS is a high-resolution instrument, however for DAR determination it has been reported to be beneficial to limit the resolution in the Orbitrap to around $35000 .{ }^{16,42,50}$ Since the resolution of individual isotopes/PTMs commonly is not necessary for DAR assignment, a resolution down to 17500 could suffice for DAR assignments. ${ }^{51}$ Cysteine conjugation commonly generates a mixture of intact ADC with an increasing portion of non-covalently linked light and heavy chains for increasing average DAR. ${ }^{52,53}$ Most publications depict light chain (LC) and heavy chains (HC) in separate spectra. However, by opting to display both LCs and HCs in the same spectrum, we could clearly observe a pronounced bias for LC in all QToF spectra (see Fig. 3) which was not observed in the Orbitrap spectra (see Fig. 4). This loss of bias mainly relates to the increased desolvation efficiency for the heavier HC species in the nano-ESI source used with the Orbitrap instrument. However, for the highest DAR species (high and max), a reversed bias for the HCs over the LCs was observed in the Orbitrap spectra, which could not be observed in any other mass spectra. The reason for this phenomenon still needs to be investigated.

Higher sensitivity can be obtained by using a nano-flow ionization source lowering the amount of sample needed for each analysis. In this study it was possible to detect down to $0.25 \mu \mathrm{g}$ of ADC with a combination of a nano-flow source and MS-detector, which is 64 times less than HIC-UV/Vis required for good spectra quality. It is worth noting that in this study the purpose of the RPLC was mainly to eliminate any off-line desalting step to save time and the chromatographic methods were therefore not run under conditions that gave a complete chromatographic resolution of the different chains. On the one hand, for RPLC-MS QToF, the flow rate was slightly higher than optimal in order to shorten the overall run time needed to analyse all batches. On the other hand, the flow rate for the RPLC-MS Orbitrap was relatively low to ensure close-to-com-

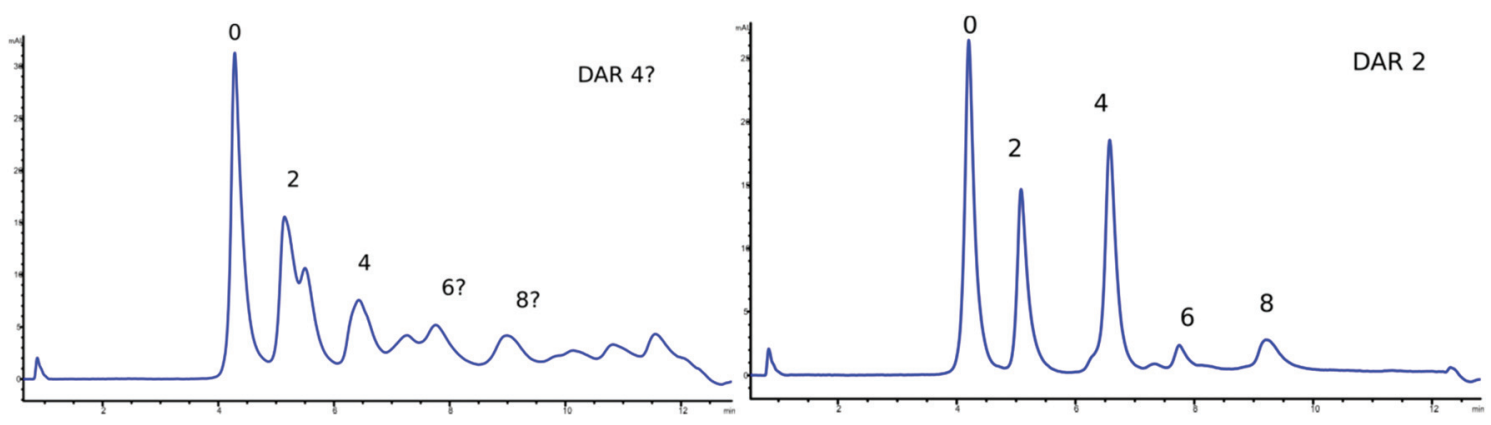

Fig. 2 HIC-UV/Vis spectrum for an early cysteine-linked ADC construct with DAR of approximately 4 (Left) and a cysteine-linked ADC construct with DAR 2 (Right) with different DAR species assigned to each peak when possible. As can be observed the annotation of DAR to specific peaks is not always straightforward even for cysteine-linked ADCs in HIC-UV/Vis. 

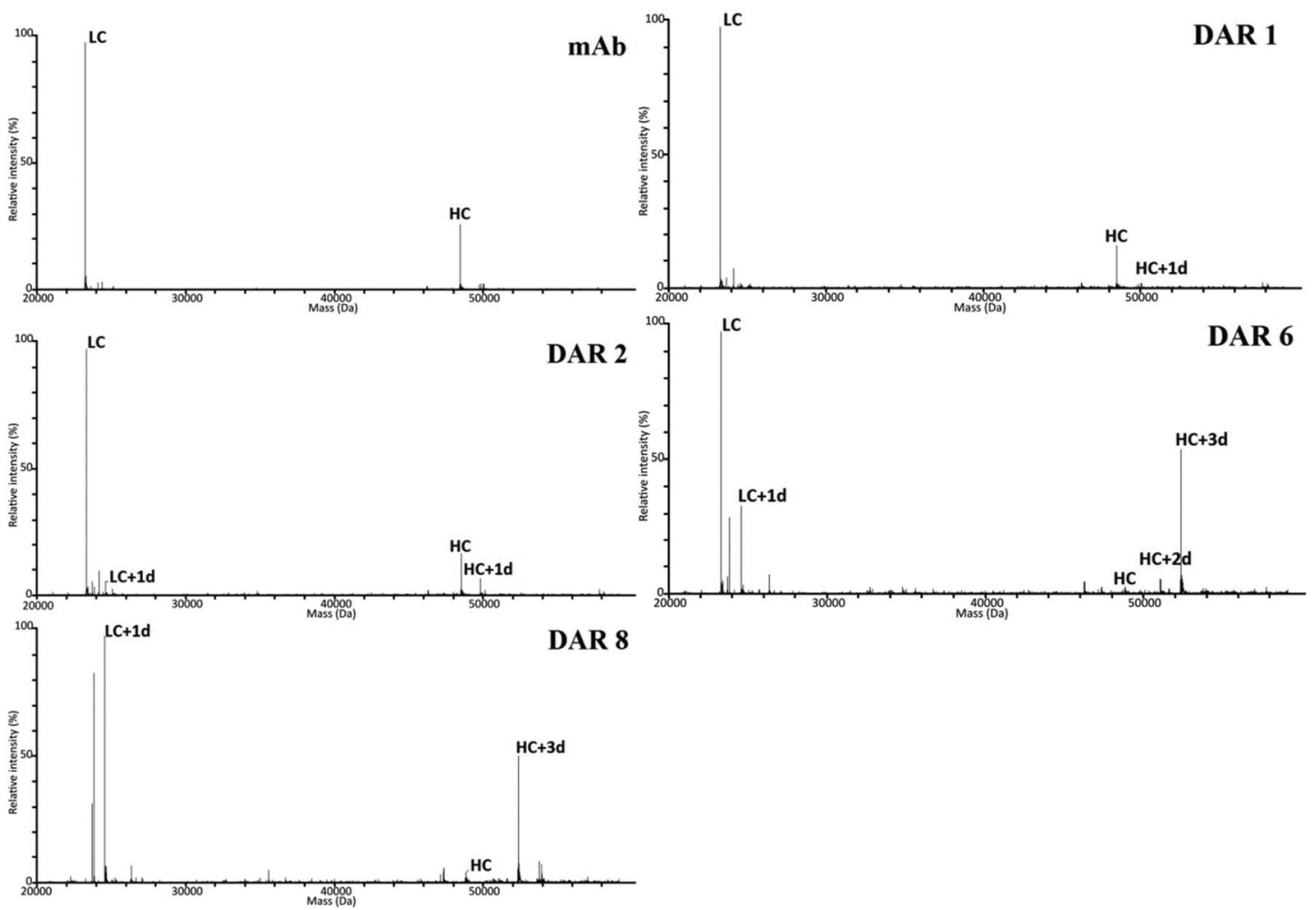

Fig. 3 RPLC-MS (QTOF) deconvoluted spectra for both light and heavy chains of non-conjugated antibody and ADCs with increasing DAR, showing the chains with different degrees of conjugation (e.g. LC with one drug attached (LC + 1d)). A bias in favour of the LC can be observed for all batches.
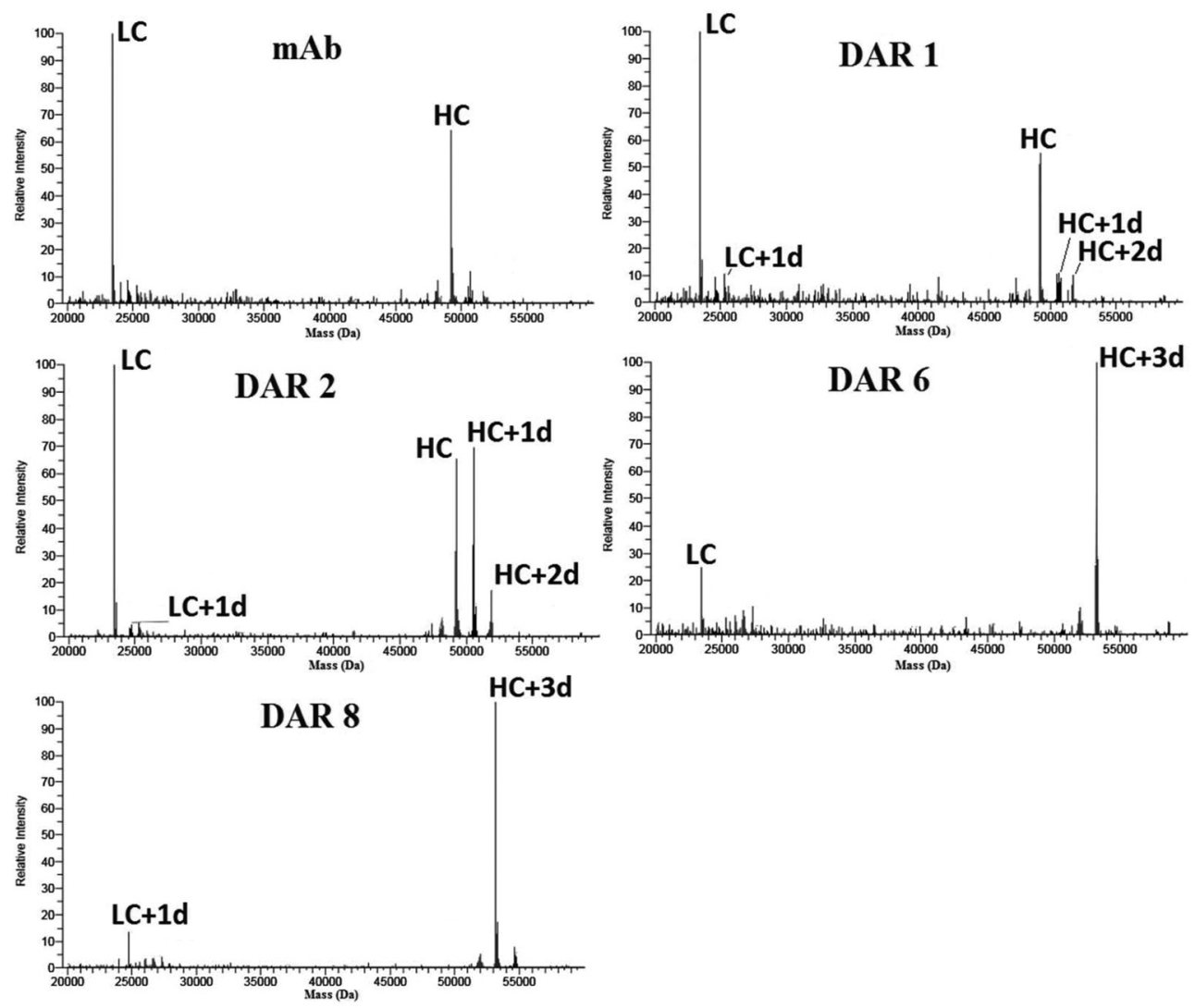

Fig. 4 RPLC-MS (Orbitrap) deconvoluted spectra of both light and heavy chains of non-conjugated antibody and ADCs with increasing DAR, showing the chains with different degrees of conjugation (e.g. LC with one drug attached (LC $+1 \mathrm{~d})$ ). 
plete desolvation of the analytes before introduction to the MS and the run time may therefore be shortened by increasing the flow rate if sufficient desolvation can be maintained. Both RPLC-MS methods used relatively low column temperatures compared to other studies ${ }^{52,54,55}$ due to the instrumental limitations for the QToF and less heat resistance of the microcolumn used for the Orbitrap. Increasing the temperature would significantly help in shortening the elution time; however, there is also a risk of on-column degradation of the analyte. Both RPLC-MS techniques showed only minor carryover effects but not as low as the HIC-UV/Vis (ESI Fig. S1-S15† for illustration with DAR $8 \mathrm{ADC}$ ) from the higher sensitivity of the MS detectors compared to the UV/Vis detector.

\section{MALDI-TOF-MS}

MALDI has less time-consuming data evaluation compared to the RPLC-MS methods since no deconvolution was needed and the elimination of a chromatographic separation step resulted in an overall quicker analysis when analysing multiple batches of the same ADC construct, as in this study. Hence, MALDI was found to be suitable for the initial screening of new potential ADC constructs. However, as can be observed in Fig. 5, all peaks had comparatively broad peak widths compared to RPLC-MS. This peak broadening is known to originate from the limited resolution of the TOF detector (in linear mode) for larger ions in combination with isotopic broadening from different PTM patterns of individual ADCs. As in
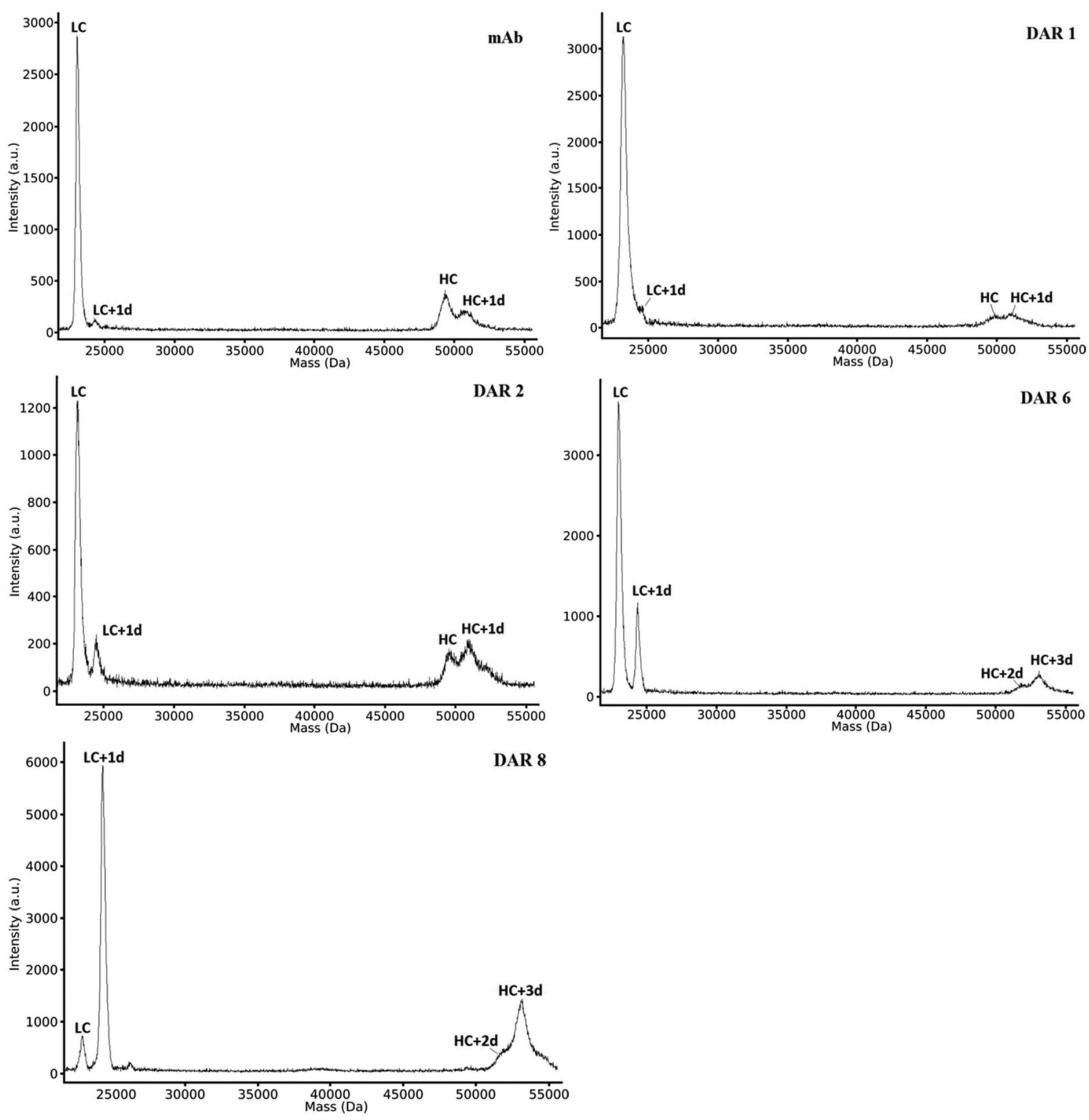

Fig. 5 MALDI-TOF-MS of both light and heavy chains of non-conjugated antibody and ADCs with increasing DAR, showing the chains with different degrees of conjugation (e.g. LC with one drug attached (LC + 1d)). Spectra for mAb, DAR 6 and 8 are acquired on a different day but from the same sample batches. Details of the sample preparation are given in the ESI and Fig. S16-S18† display additional spectra. 
Table 2 Average molecular weight in triplicate of deglycosylated and reduced mAb and ADC samples for both non-conjugated chains (LC and HC) and with different drug loads (e.g. LC with one drug attached (LC $+1 \mathrm{~d})$ ) with the RSD of each mass in parentheses

\begin{tabular}{lllll}
\hline & MALDI-TOF-MS & RPLC-MS(QToF) & RPLC-MS(Orbitrap) & Theoretical mass $^{a}$ \\
\hline LC & $23047(0.020 \%)$ & $23444(0.0025 \%)$ & $23456(0.002 \%)$ & $23440 \mathrm{Da}$ \\
LC + 1d & $24366(0.006 \%)$ & $24760(0.0002 \%)$ & $24773(0.002)$ & $24767 \mathrm{Da}$ \\
HC & $49730(0.115 \%)$ & $49159(0.0022 \%)$ & $49321(0.071 \%)$ & $49300 \mathrm{Da}$ \\
HC + 1d & $51338(0.196 \%)$ & $50473(0.0009 \%)$ & $50575(0.052 \%)$ & $50617 \mathrm{Da}$ \\
HC + 2d & $52301(0.201 \%)$ & $51792(0.0004 \%)$ & $51839(0.025 \%)$ & $51934 \mathrm{Da}$ \\
HC + 3d & $53463(0.112 \%)$ & $53108(0.0003 \%)$ & $53099(0.002 \%)$ & $53251 \mathrm{Da}$
\end{tabular}

${ }^{a}$ Theoretical masses are calculated from sequences at drugbank.com and from 10.1021/acs.analchem.5b04561.

RPLC-MS, we could observe a bias for the light chains over the heavy chains. This bias was surprisingly prominent for the non-conjugated antibodies. In MALDI, the bias for LC fragments was extra prominent as certain locations on certain spots only gave a signal for the light chain. Thus, the assessment of the DAR value was dependent on the laser spot location and summing up only "sweet spots" that included the signal for the heavy chains. This could potentially impact the DAR calculation since most of the drugs for cysteine linked ADCs are linked to the heavy chains. However, as seen in Table 1, MALDI produced DAR values that compared well to HIC-UV/Vis and RPLC-MS.

One aspect that required extra attention for MALDI was that sample application deviations also influenced the data. On comparing different sample application techniques in early trials, it became evident that samples that differentiated visually after crystallization (in amount and shape of crystals formed) had significantly different qualities of the obtained spectra (data not shown). An automation of the sample preparation which would eliminate human errors could thus further improve the reproducibility. In our case, the sandwich deposition technique gave the most reproducible results and was therefore chosen over the dried-drop deposition, despite having overall lower signal intensity.

\section{Mass accuracy}

The molecular weight $\left(M_{\mathrm{w}}\right)$ of the different chains from all MS based techniques can be found in Table 2. As predicted, the highest divergence in these values was observed for the MALDI-TOF-MS technique (see Table 2 and ESI Table S1 $\dagger$ ). However, by using the peak intensity instead of the area for the MS based techniques, the average DAR values for the MALDI-TOF-MS coincided well with what was observed in the other techniques for most batches (see Table 1). Another noteworthy observation was that despite the RPLC-MS (QToF) having the lowest RSD values, the Mw differed further from the theoretical values than those determined by the Orbitrap (ESI, Tables S2 and S3 $\dagger$ ), in particular for the heavy chains which are all about $140 \mathrm{Da}$ smaller than their theoretical values. Why this discrepancy was seen for the QToF-based analysis only has yet to be investigated. Another trend observed for all techniques was that the mass shift upon conjugation for the heavy chains differed to that observed for the light chains, despite low standard deviations of the detected molecular weights for both chains (see ESI, Table S4†). The reason for this discrepancy remains elusive.

\section{Conclusions}

Despite the clear differences in assigned molecular weight accuracy, all tested techniques (including HIC-UV/Vis) provided satisfactory DAR results for the ADC analysis. However, a combination of at least two complementary techniques is recommended to verify the DAR values, especially for ADCs with a low average DAR. Depending on the required level of mass accuracy, MALDI-TOF-MS could be a useful option due to its simplicity in both sample preparation and data evaluation. To our knowledge, we are the first to evaluate MALDI's potential use for DAR determination on disulphide bond reduced ADC samples. The shorter time requirement compared to RPLC-MS from sample preparation to reported DAR value makes it a compelling option in the early stages of the ADC discovery phase where many different batches need to be analysed swiftly to verify the success rate of different synthetic protocols. Apart from the small system volumes of the LC-system used and high sensitivity of the Orbitrap system, we could not report a major advantage of using a high-resolution MS such as an Orbitrap as the detector for RPLC-MS for DAR determination. With more techniques proven to aid in ADC analysis, this field is far from finished evolving and there are still many challenges ahead. ${ }^{56}$ However, our hope is that this publication can serve as a guide especially for new labs starting up in this constantly growing research field.

\section{Author contributions}

All authors have given approval to the final version of the manuscript.

\section{Conflicts of interest}

There are no conflicts to declare. 


\section{Acknowledgements}

The authors would like to acknowledge the Swedish institute for Strategic Research ID14-0081, the Swedish Research Council SRC 2015-4870 (JB) and Carl Trygger Foundation (CTS 15:57) (SBL) for the financial support for this study.

\section{References}

1 A. Beck, T. Wurch, C. Bailly and N. Corvaia, Strategies and Challenges for the next Generation of Therapeutic Antibodies, Nat. Rev. Immunol., 2010, 10(5), 345-352.

2 V. Chudasama, A. Maruani and S. Caddick, Recent Advances in the Construction of Antibody-drug Conjugates, Nat. Chem., 2016, 8(2), 114-119.

3 C. Chalouni and S. Doll, Fate of Antibody-Drug Conjugates in Cancer Cells, J. Exp. Clin. Cancer Res., 2018, 37(1), 20.

4 N. Joubert, C. Denevault-Sabourin, F. Bryden and M. C. Viaud-Massuard, Towards Antibody-Drug Conjugates and Prodrug Strategies with Extracellular Stimuli-Responsive Drug Delivery in the Tumor Microenvironment for Cancer Therapy, Eur. J. Med. Chem., 2017, 393-415.

5 J. R. McCombs and S. C. Owen, Antibody Drug Conjugates: Design and Selection of Linker, Payload and Conjugation Chemistry, AAPS J., 2015, 17(2), 339-351.

6 A. S. Rosenberg, Effects of Protein Aggregates: An Immunologic Perspective, AAPS J., 2006, 8(3), E501-E507.

7 H. Schellekens, J. S. Smolen, M. Dicato and R. M. Rifkin, Safety and Efficacy of Biosimilars in Oncology, Lancet Oncol., 2016, 17(11), e502-e509.

8 S. Rosati, N. J. Thompson, A. J. R. Heck, S. Rosati, N. J. Thompson and A. J. R. Heck, Tackling the Increasing Complexity of Therapeutic Monoclonal Antibodies with Mass Spectrometry, Trends Anal. Chem., 2013, 48, 72-80.

9 P. M. Liu, L. Zou, C. Sadhu, W. D. Shen and S. Nock, Comparative Immunogenicity Assessment: A Critical Consideration for Biosimilar Development, Bioanalysis, 2015, 7(3), 373-381.

10 US Food and Drug administration (FDA), Guidance for Industry- Monoclonal Antibodies Used as Reagents in Drug Manufacturing https://www.fda.gov/downloads/ BiologicsBloodVaccines/GuidanceComplianceRegulatory Information/Guidances/Blood/ucm080417.pdf.

11 US Food and Drug administration (FDA), Assay Development and Validation for Immunogenicity Testing of Therapeutic Protein Products - Guidance for Industry http://www.fda.gov/Drugs/GuidanceComplianceRegulatory Information/Guidances/default.htm0A, http:/www.fda.gov/ BiologicsBloodVaccines/GuidanceComplianceRegulatory Information/Guidances/default.htm0A, http:/www.fda. gov/MedicalDevices/DeviceRegulationandGuidanc.

12 J. Lu, F. Jiang, A. Lu and G. Zhang, Linkers Having a Crucial Role in Antibody-Drug Conjugates, Int. J. Mol. Sci., 2016, $17(12), 561$.
13 S. C. Alley, D. R. Benjamin, S. C. Jeffrey, N. M. Okeley, D. L. Meyer, R. J. Sanderson and P. D. Senter, Contribution of Linker Stability to the Activities of Anticancer Immunoconjugates, Bioconjugate Chem., 2008, 19(3), 759-765.

14 A. E. Albers, A. W. Garofalo, P. M. Drake, R. Kudirka, G. W. de Hart, R. M. Barfield, J. Baker, S. Banas and D. Rabuka, Exploring the Effects of Linker Composition on Site-Specifically Modified Antibody-drug Conjugates, Eur. J. Med. Chem., 2014, 88, 3-9.

15 H. D. King, G. M. Dubowchik, H. Mastalerz, D. Willner, S. J. Hofstead, R. A. Firestone, S. J. Lasch and P. A. Trail, Monoclonal Antibody Conjugates of Doxorubicin Prepared with Branched Peptide Linkers: Inhibition of Aggregation by Methoxytriethyleneglycol Chains, J. Med. Chem., 2002, 45(19), 4336-4343.

16 F. Debaene, A. Bœuf, E. Wagner-Rousset, O. Colas, D. Ayoub, N. Corvaïa, A. Van Dorsselaer, A. Beck and S. Cianférani, Innovative Native MS Methodologies for Antibody Drug Conjugate Characterization: High Resolution Native MS and IM-MS for Average DAR and DAR Distribution Assessment, Anal. Chem., 2014, 86(21), 1067410683.

17 R. Y.-C. Huang, E. G. Deyanova, D. Passmore, V. Rangan, S. Deshpande, A. A. Tymiak and G. Chen, Utility of Ion Mobility Mass Spectrometry for Drug-to-Antibody Ratio Measurements in Antibody-Drug Conjugates, J. Am. Soc. Mass Spectrom., 2015, 26(10), 1791-1794.

18 J. Chen, S. Yin, Y. Wu and J. Ouyang, Development of a Native Nanoelectrospray Mass Spectrometry Method for Determination of the Drug-to-Antibody Ratio of Antibody-Drug Conjugates, Anal. Chem., 2013, 85(3), 1699-1704.

19 K. J. Pacholarz and P. E. Barran, Use of a Charge Reducing Agent to Enable Intact Mass Analysis of Cysteine-Linked Antibody-Drug-Conjugates by Native Mass Spectrometry, EuPa Open Proteomics, 2016, 11, 23-27.

20 J. F. Valliere-Douglass, W. A. McFee and O. Salas-Solano, Native Intact Mass Determination of Antibodies Conjugated with Monomethyl Auristatin E and $\mathrm{F}$ at Interchain Cysteine Residues, Anal. Chem., 2012, 84(6), 2843-2849.

21 I. D. G. Campuzano, C. Netirojjanakul, M. Nshanian, J. L. Lippens, D. P. A. Kilgour, S. L. Van Orden and J. Loo, Native-MS Analysis of Monoclonal Antibody Conjugates by Fourier Transform Ion Cyclotron Resonance Mass Spectrometry, Anal. Chem., 2018, 90, 745-751.

22 R. E. Birdsall, S. M. McCarthy, M. C. Janin-Bussat, M. Perez, J.-F. Haeuw, W. Chen and A. Beck, A Sensitive Multidimensional Method for the Detection, Characterization, and Quantification of Trace Free Drug Species in Antibody-Drug Conjugate Samples Using Mass Spectral Detection, $m A b s, 2016,8(2), 306-317$.

23 P. L. Ross and J. L. Wolfe, Physical and Chemical Stability of Antibody Drug Conjugates: Current Status, J. Pharm. Sci., 2016, 105(2), 391-397. 
24 J. Guo, S. Kumar, A. Prashad, J. Starkey and S. K. Singh, Assessment of Physical Stability of an Antibody Drug Conjugate by Higher Order Structure Analysis: Impact of Thiol-Maleimide Chemistry, Pharm. Res., 2014, 31(7), 17101723.

25 A. Ehkirch, V. D’Atri, F. Rouviere, O. Hernandez-Alba, A. Goyon, O. Colas, M. Sarrut, A. Beck, D. Guillarme, S. Heinisch, et al., An Online Four-Dimensional HIC $\times$ SEC-IM $\times$ MS Methodology for Proof-of-Concept Characterization of Antibody Drug Conjugates, Anal. Chem., 2018, 90(3), 1578-1586.

26 N. S. Beckley, K. P. Lazzareschi, H. W. Chih, V. K. Sharma and H. L. Flores, Investigation into Temperature-Induced Aggregation of an Antibody Drug Conjugate, Bioconjugate Chem., 2013, 24(10), 1674-1683.

27 T. Chen, Y. Chen, C. Stella, C. D. Medley, J. A. Gruenhagen and K. Zhang, Antibody-Drug Conjugate Characterization by Chromatographic and Electrophoretic Techniques, J. Chromatogr. B: Anal. Technol. Biomed. Life Sci., 2016, 1032, 39-50.

28 R. Neupane, M. Källsten, F. Lehmann and J. Bergquist, Effect of Mobile Phase Composition on the Analysis of Aggregates of Antibody Drug Conjugates (ADCs) Using Size Exclusion Chromatography, Anal. Methods, 2018, 10(9), 938-941.

29 S. M. Hengel, R. Sanderson, J. Valliere-Douglass, N. Nicholas, C. Leiske and S. C. Alley, Measurement of in Vivo Drug Load Distribution of Cysteine-Linked Antibody-Drug Conjugates Using Microscale Liquid Chromatography Mass Spectrometry, Anal. Chem., 2014, 86(7), 3420-3425.

30 S. Rosati, R. J. Rose, N. J. Thompson, E. Van Duijn, E. Damoc, E. Denisov, A. Makarov and A. J. R. Heck, Exploring an Orbitrap Analyzer for the Characterization of Intact Antibodies by Native Mass Spectrometry, Angew. Chem., Int. Ed., 2012, 51(52), 12992-12996.

31 N. Said, R. Gahoual, L. Kuhn, A. Beck, Y.-N. François and E. Leize-Wagner, Structural Characterization of Antibody Drug Conjugate by a Combination of Intact, Middle-up and Bottom-up Techniques Using Sheathless Capillary Electrophoresis - Tandem Mass Spectrometry as nanoESI Infusion Platform and Separation Method, Anal. Chim. Acta, 2016, 918, 50-59.

32 V. D’Atri, S. Fekete, D. Stoll, M. Lauber, A. Beck and D. Guillarme, Characterization of an Antibody-Drug Conjugate by Hydrophilic Interaction Chromatography Coupled to Mass Spectrometry, J. Chromatogr. B: Anal. Technol. Biomed. Life Sci., 2018, 1080(June 2017), 37-41.

33 A. Cusumano, D. Guillarme, A. Beck and S. Fekete, Practical Method Development for the Separation of Monoclonal Antibodies and Antibody-Drug-Conjugate Species in Hydrophobic Interaction Chromatoraphy, Part 2: Optimization of the Phase System, J. Pharm. Biomed. Anal., 2016, 121, 161-173.

34 S. Fekete, J.-L. Veuthey and D. Guillarme, New Trends in Reversed-Phase Liquid Chromatographic Separations of
Therapeutic Peptides and Proteins: Theory and Applications, J. Pharm. Biomed. Anal., 2012, 69, 9-27.

35 R. Neupane and J. Bergquist, Analytical Techniques for the Characterization of Antibody Drug Conjugates: Challenges and Prospects, Eur. J. Mass Spectrom., 2017, 23(6), 417-426.

36 L. N. Le, J. M. R. Moore, J. Ouyang, X. Chen, M. D. H. Nguyen and W. J. Galush, Profiling Antibody Drug Conjugate Positional Isomers: A System-of-Equations Approach, Anal. Chem., 2012, 84(17), 7479-7486.

37 D. Firth, L. Bell, M. Squires, S. Estdale and C. McKee, A Rapid Approach for Characterization of Thiol-Conjugated Antibody-drug Conjugates and Calculation of Drug-antibody Ratio by Liquid Chromatography Mass Spectrometry, Anal. Biochem., 2015, 485, 34-42.

38 J. A. Francisco, cAC10-vcMMAE, an Anti-CD30-Monomethyl Auristatin E Conjugate with Potent and Selective Antitumor Activity, Blood, 2003, 102(4), 1458-1465.

39 J. Valliere-Douglass, A. Wallace and A. Balland, Separation of Populations of Antibody Variants by Fine Tuning of Hydrophobic-Interaction Chromatography Operating Conditions, J. Chromatogr. A, 2008, 1214(1-2), 81-89.

40 J. A. Queiroz, C. T. Tomaz and J. M. S. Cabral, Hydrophobic Interaction Chromatography of Proteins, J. Biotechnol., 2001, 87, 143-159.

41 L. Ducry, Antibody-Drug Conjugates, in Methods in Molecular Biology, ed. L. Ducry, Humana Press, Totowa, NJ, 2013, vol. 1045.

42 X. Zhang, Characterization of Monoclonal Antibodies and ADCs Using a Benchtop Orbitrap Mass Spectrometer Characterization of Monoclonal Antibodies and ADCs Using a Ben Characterization of Monoclonal Antibodies and ADCs Using a Ben Xiaoxi Benchtop Orbitrap Mass Spec. In Poster note 64397; Thermo Fisher Scientific, 2015.

43 M. M. Siegel, I. J. Hollander, P. R. Hamann, J. P. James, L. Hinman, B. J. Smith, A. P. Farnsworth, A. Phipps, D. J. King and M. Karas, Matrix-Assisted UV-Laser Desorption/ionization Mass Spectrometric Analysis of Monoclonal Antibodies for the Determination of Carbohydrate, Conjugated Chelator, and Conjugated Drug Content, Anal. Chem., 1991, 63(21), 2470-2481.

44 J. F. Valliere-Douglass, S. M. Hengel and L. Y. Pan, Approaches to Interchain Cysteine-Linked ADC Characterization by Mass Spectrometry, Mol. Pharm., 2015, 12(6), 1774-1783.

45 R. Y. C. Huang and G. Chen, Characterization of Antibody Drug Conjugates by Mass Spectrometry: Advances and Future Trends, Drug Discovery Today, 2016, 21(5), 850-855.

46 A. Beck, G. Terral, F. Debaene, E. Wagner-Rousset, J. Marcoux, M.-C. Janin-Bussat, O. Colas, A. Dorsselaer and S. Van Cianférani, Cutting-Edge Mass Spectrometry Methods for the Multi-Level Structural Characterization of Antibody-Drug Conjugates, Expert Rev. Proteomics, 2016, 13(2), 157-183.

47 O. V. Friese, J. N. Smith, P. W. Brown and J. C. Rouse, Practical Approaches for Overcoming Challenges in Heightened Characterization of Antibody-Drug Conjugates with New 
Methodologies and Ultrahigh-Resolution Mass Spectrometry. mAbs, Taylor \& Francis, 2018, pp. 1-11.

48 D. Boyd, T. Kaschak and B. Yan, HIC Resolution of an IgG1 with an Oxidized Trp in a Complementarity Determining Region, J. Chromatogr. B: Anal. Technol. Biomed. Life Sci., 2011, 879(13-14), 955-960.

49 M. M. C. Sun, K. S. Beam, C. G. Cerveny, K. J. Hamblett, R. S. Blackmore, M. Y. Torgov, F. G. M. Handley, N. C. Ihle, P. D. Senter and S. C. Alley, Reduction-Alkylation Strategies for the Modification of Specific Monoclonal Antibody Disulfides, Bioconjugate Chem., 2005, 16(5), 1282-1290.

50 S. Rosati, E. T. J. van den Bremer, J. Schuurman, P. W. H. I. Parren, J. P. Kamerling and A. J. R. Heck, In-Depth Qualitative and Quantitative Analysis of Composite Glycosylation Profiles and Other Micro-Heterogeneity on Intact Monoclonal Antibodies by High-Resolution Native Mass Spectrometry Using a Modified Orbitrap, $m A b s, 2013$, 5(6), 917-924.

51 J. Marcoux, T. Champion, O. Colas, E. Wagner-Rousset, N. Corvaïa, A. Van Dorsselaer, A. Beck and S. Cianférani, Native Mass Spectrometry and Ion Mobility Characterization of Trastuzumab Emtansine, a LysineLinked Antibody Drug Conjugate, Protein Sci., 2015, 24(8), 1210-1223.
52 R. E. Birdsall, H. Shion, F. W. Kotch, A. Xu, T. J. Porter and W. Chen, A Rapid on-Line Method for Mass Spectrometric Confirmation of a Cysteine-Conjugated Antibody-DrugConjugate Structure Using Multidimensional Chromatography, mAbs, 2015, 7(6), 1036-1044.

53 B. Wiggins, L. Liu-Shin, H. Yamaguchi and G. Ratnaswamy, Characterization of Cysteine-Linked Conjugation Profiles of Immunoglobulin G1 and Immunoglobulin G2 AntibodyDrug Conjugates, J. Pharm. Sci., 2015, 104(4), 1362-1372.

54 C. Lanshoeft, S. Cianférani and O. Heudi, Generic Hybrid Ligand Binding Assay Liquid Chromatography HighResolution Mass Spectrometry-Based Workflow for Multiplexed Human Immunoglobulin G1 Quantification at the Intact Protein Level: Application to Preclinical Pharmacokinetic Studies, Anal. Chem., 2017, 89(4), 26282635.

55 E. Wagner-Rousset, M.-C. Janin-Bussat, O. Colas, M. Excoffier, D. Ayoub, J.-F. Haeuw, I. Rilatt, M. Perez, N. Corvaïa and A. Beck, Antibody-Drug Conjugate Model Fast Characterization by LC-MS Following IdeS Proteolytic Digestion, $m A b s, 2014,6(1), 173-184$.

56 A. Wagh, H. Song, M. Zeng, L. Tao and T. K. Das, Challenges and New Frontiers in Analytical Characterization of Antibody-Drug Conjugates, $m A b s, 2018$, 10(2), 222-243. 